\title{
Comparison of Exact Solutions for Heat Transfer in Extended Surfaces of Different Geometries
}

\author{
K. J. Moleofane and R. J. Moitsheki \\ Center for Differential Equations, Continuum Mechanics and Applications, School of Computational and Applied Mathematics, \\ University of the Witwatersrand (Wits), Private Bag 3, Johannesburg 2050, South Africa
}

Correspondence should be addressed to R. J. Moitsheki; raseelo.moitsheki@wits.ac.za

Received 17 January 2014; Accepted 19 February 2014; Published 19 March 2014

Academic Editor: Mariano Torrisi

Copyright (C) 2014 K. J. Moleofane and R. J. Moitsheki. This is an open access article distributed under the Creative Commons Attribution License, which permits unrestricted use, distribution, and reproduction in any medium, provided the original work is properly cited.

\begin{abstract}
We consider a steady state problem for heat transfer in fins of various geometries, namely, rectangular, radial, and spherical. The nonlinear steady state problem is linearizable provided that the thermal conductivity is the differential consequence of the term involving the heat transfer coefficient. As such, one is able to construct exact solutions. On the other hand, we employ the Lie point symmetry methods when the problem is not linearizable. Some interesting results are obtained and analyzed. The effects of the parameters such as thermogeometric fin parameter and the exponent on temperature are studied. Furthermore, fin efficiency and heat flux along the fin length of a spherical geometry are also studied.
\end{abstract}

\section{Introduction}

Heat transfer rate from a hot body to the surrounding may be increased by surfaces which extended into that surrounding. These extended surfaces are referred to as fins. Extended surfaces are found in many engineering appliances. Thus, mathematical modeling of the heat transfer through these surfaces and the solution are of continued interest. The heat transfer in fins is governed by boundary value problems (BVPs) which are rendered highly nonlinear by the dependency of thermal properties on temperature. In this study, both the heat transfer coefficient and thermal conductivity are given as a power law function of temperature.

The interest in solutions of fin problems continues unabated. Many symmetry analysts [1-5] analysed the fin equation when the heat transfer coefficient is given as a function of a space variable. Such a function was classified by direct methods [2] and the extended analysis was done in [4]. Only general solutions were provided in this case. It was claimed that exact solutions of steady fin problems exist only when thermal conductivity and heat transfer coefficients are constants [6]. However Moitsheki et al. [7] have shown that solutions may exist even whenthese thermal parame- ters are temperature dependent. In recent years Moitsheki $[8,9]$ and Moitsheki and Mhlongo [10] constructed exact solutions for the convective heat transfer in fins of different profiles. Furthermore, Ndlovu and Moitsheki [11] provided the approximate analytical solutions to steady state heat transfer in fins of different profiles which could not be solved exactly. In their studies an excellent comparison between exact and approximate solutions was established. One may also refer to the work by Moradi [12] and many other scholars.

In this study, we consider heat conduction problem in fins of different geometries and in particular the spherical fin which has never been studied before. We compare the exact solutions of heat transfer in rectangular, radial, and spherical fins. We further compare the fin efficiencies and effectiveness of these fins and determine the effects of thermal parameters in a spherical fin. This paper is arranged as follows. In Section 2, we present the description of the models considered. In Section 3 we briefly discuss the Lie point symmetry methods. Following linearization, the exact solution is provided in Section 4. In Section 5, we analyze the problem when linearization fails. Conclusions are provided in Section 6. 


\section{Mathematical Description of a Fin Problem}

We consider a fin of an arbitrary geometry with the length (or radius) $R$ and a cross-sectional area $A_{c}$. The perimeter of the fin is given by $P$. The fin is attached to a fixed prime surface of temperature $T_{b}$ and extends to an ambient fluid of temperature $T_{a}$. The energy balance equation is given by

$$
\frac{A_{c}}{R^{\alpha}} \frac{d}{d R}\left[R^{\alpha} K(T) \frac{d T}{d R}\right]=P H(T)\left(T-T_{a}\right),
$$

and the relevant boundary conditions are

$$
T\left(R_{0}\right)=T_{b},\left.\quad \frac{d T}{d R}\right|_{R=R_{1}}=0 .
$$

The Greek letter $\alpha$ represents different geometries; for example $\alpha=0,1$, and 2 represent the longitudinal rectangular, the rectangular radial, and the spherical fin as shown in Figures 1,2 , and 3 .

Introducing the nondimensional variables and numbers,

$$
\begin{aligned}
\theta=\frac{T-T_{a}}{T_{b}-T_{a}}, \quad r=\frac{R-R_{1}}{R_{0}-R_{1}}, & H=h_{b}\left(\frac{T-T_{a}}{T_{b}-T_{a}}\right)^{n}, \\
K=k_{a}\left(\frac{T-T_{a}}{T_{b}-T_{a}}\right)^{m}, & M^{2}=\frac{P h_{b} L^{2}}{k_{a} A_{c}},
\end{aligned}
$$

and then (1) and the boundary conditions (2) become

$$
\begin{gathered}
\frac{1}{r^{\alpha}} \frac{d}{d r}\left[r^{\alpha} \theta^{m} \frac{d \theta}{d r}\right]-M^{2} \theta^{n+1}=0, \quad 0 \leq r \leq 1, \\
\theta(1)=1, \quad \theta^{\prime}(0)=0 .
\end{gathered}
$$

Two main cases may be analyzed, namely, $m=n$ and $m \neq n$. One may construct exact solution when $m=n$ since the problem is linearizable and employ symmetry methods given $m \neq n$.

\section{A brief Account on Lie Point Symmetry Analysis}

In this section we provide a brief theory of Lie point symmetry techniques. In short, a symmetry of a differential equation is an invertible transformation of the dependent and independent variables that does not change the original differential equation. Detailed theory and applications of Lie symmetry groups may be found in the texts such as those of [13-17]. Since in this study we deal with nonlinear second order ODEs therefore we will restrict our discussion to the determination of symmetries for such equations.

We seek transformations of the form

$$
\bar{r}=r+\xi(r, \theta) a, \quad \bar{y}=y+\eta(r, \theta) a,
$$

called the infinitesimal transformations generated by the vector

$$
X=\xi(r, \theta) \frac{\partial}{\partial r}+\eta(r, \theta) \frac{\partial}{\partial \theta}
$$

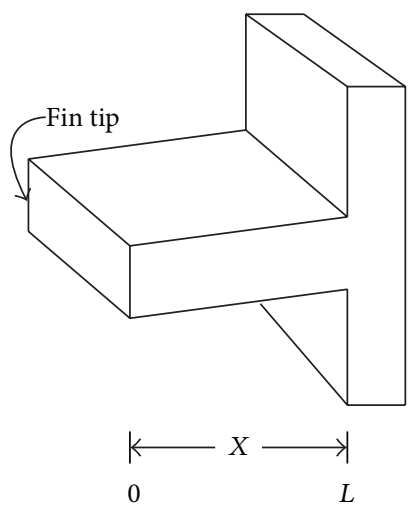

FIGURE 1: Graphical representation of a longitudinal rectangular fin.

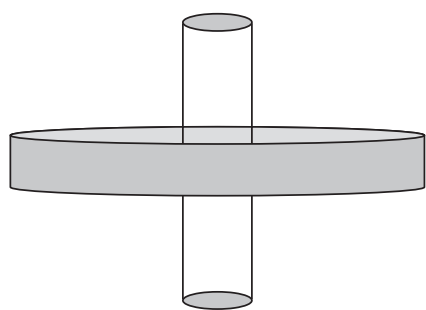

FIGURE 2: Graphical representation of a rectangular radial fin.

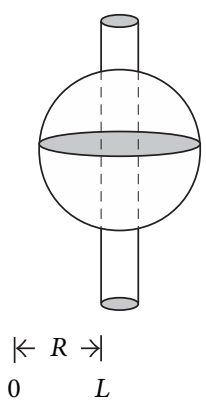

FIGURE 3: Graphical representation of a spherical fin.

which leave the given differential equation invariant. Here $a$ is a group parameter. The group generated by transformations (6) is called a one-parameter group of transformations. If the given equation is of second order, for instance,

$$
f\left(r, \theta, \theta^{\prime}, \theta^{\prime \prime}\right)=0
$$

then we extend the symmetry generator (7) to

$$
X^{[2]}=\xi \frac{\partial}{\partial r}+\eta \frac{\partial}{\partial \theta}+\zeta_{1} \frac{\partial}{\partial \theta^{\prime}}+\zeta_{2} \frac{\partial}{\partial \theta^{\prime \prime}},
$$

where

$$
\begin{aligned}
& \zeta_{1}=D(\eta)-\theta^{\prime} D(\xi), \\
& \zeta_{2}=D\left(\zeta_{1}\right)-\theta^{\prime \prime} D(\xi),
\end{aligned}
$$

with $D$ being the total derivative operator defined as

$$
D=\frac{\partial}{\partial r}+\theta^{\prime} \frac{\partial}{\partial \theta}+\theta^{\prime \prime} \frac{\partial}{\partial \theta^{\prime}}+\cdots
$$




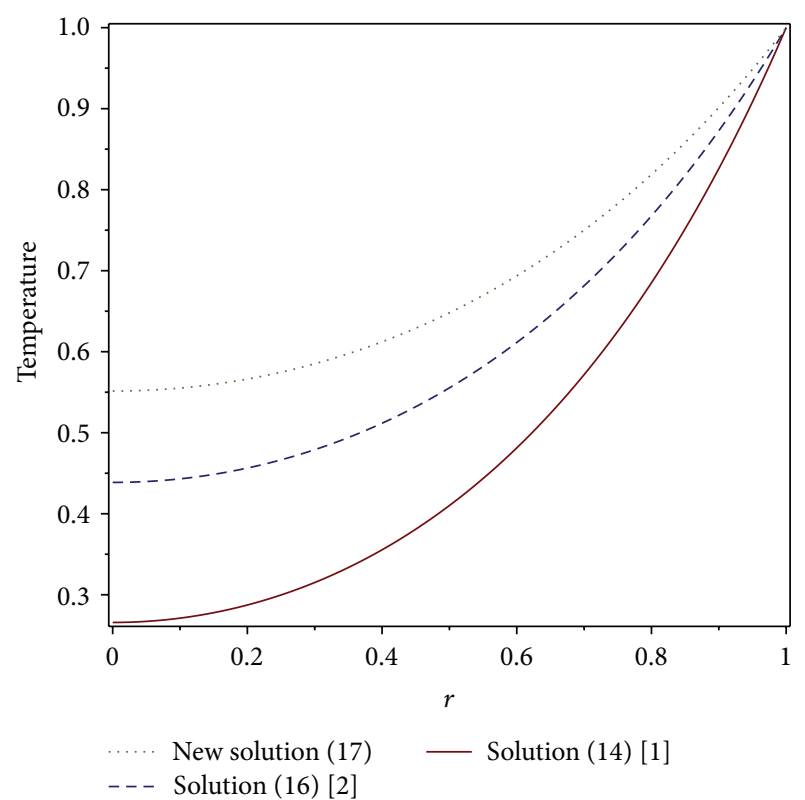

FIgURE 4: Temperature profile given solutions (14), (16), and (17). Here $M=2$ and $n=0$.

The invariance surface condition

$$
X^{[2]}\left(\text { Equation (8)) }\left.\right|_{(\text {Equation (8)) }}=0\right.
$$

yields the overdetermined system of linear equations called the determining equation which may be solved to obtain the admitted symmetry generators (or equivalently symmetry transformation groups). In our analysis we determine the symmetries admitted by the single governing equation rather than the boundary value problem (BVP). Usually the symmetry algebra for the BVP is less in dimensions than that of the governing equation (see also [13]).

\section{Linearization and Exact Solutions}

It has been proven in [9] that equation such as (4) is linearizable provided that $m=n$. Thus assuming $m=n$ and letting $y=\theta^{n+1}$ then (4)

$$
\frac{d^{2} y}{d r^{2}}+\frac{\alpha}{r} \frac{d y}{d r}-(n+1) M^{2} y=0
$$

Several subcases arise, namely, $\alpha=0,1,2$ and arbitrary, given $n<1$ and $n>1$.

Case $1(\alpha=0, n<-1$ and $n>-1)$. This case has been solved in [7]. In this case the solutions are given by

$$
\begin{aligned}
& \theta=\left\{\frac{\cosh (\sqrt{n+1} M r)}{\cosh (\sqrt{n+1} M)}\right\}^{1 /(n+1)}, \quad-1<n<\infty, \\
& \theta=\left\{\frac{\sinh (\sqrt{n+1} M r)}{\sinh (\sqrt{n+1} M)}\right\}^{1 /(n+1)}, \quad-1<n<0 .
\end{aligned}
$$

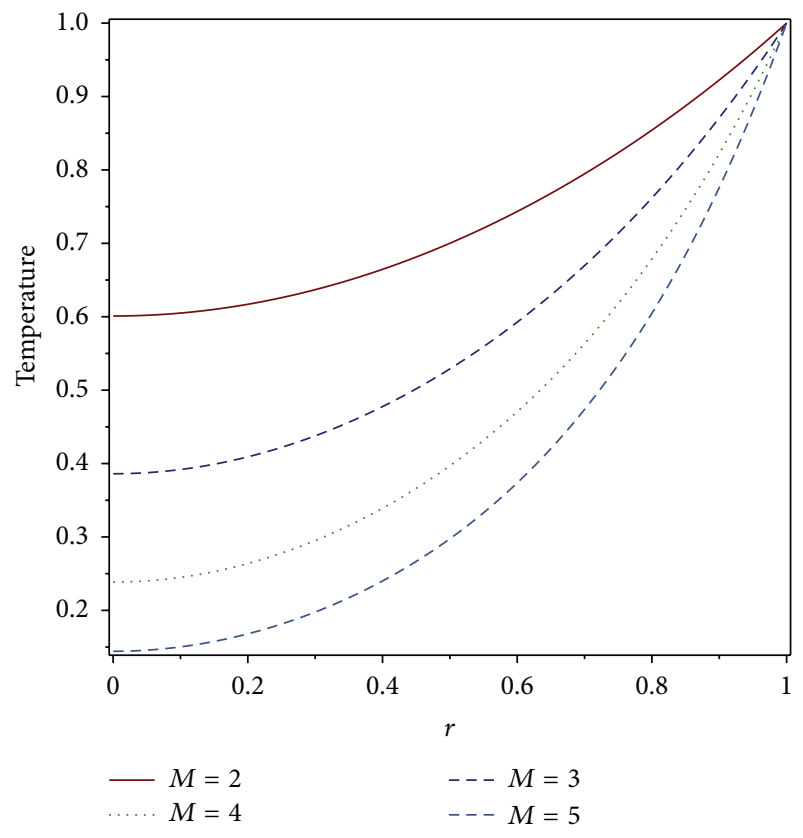

FIGURE 5: Temperature profile given solutions (17) for varying values of $M$. Here $n=2$ is fixed.

The solution for $n<-1$ is given in terms of sine and cosine functions.

Case $2(\alpha=1, n<-1$ and $n>-1)$. This case has been solved in [8]. In this case the exact solutions are given:

$$
\theta=\left\{\frac{I_{0}(\sqrt{n+1} M r)}{I_{0}(\sqrt{n+1} M)}\right\}^{1 /(n+1)}, \quad-1<n<\infty .
$$

The solutions for $n<-1$ are given in terms of Bessel functions.

Case $3(\alpha=2, n<-1$ and $n>-1)$. In this case we obtain the exact solutions

$$
\begin{aligned}
& \theta=\left\{\frac{1}{r}\left[\frac{\sinh (M \sqrt{n+1} r)}{\sinh (M \sqrt{n+1})}\right]\right\}^{1 /(n+1)}, \quad n>-1, \\
& \theta=\left\{\frac{1}{r}\left[\frac{\sin (M \sqrt{n+1} r)}{\sin (M \sqrt{n+1})}\right]\right\}^{1 /(n+1)}, \quad n<-1 .
\end{aligned}
$$

The solutions (14), (16), and (17) are depicted in Figure 4. Figures 5 and 6 depict the plot of solution (17) for varying $M$ and $n$, respectively. 


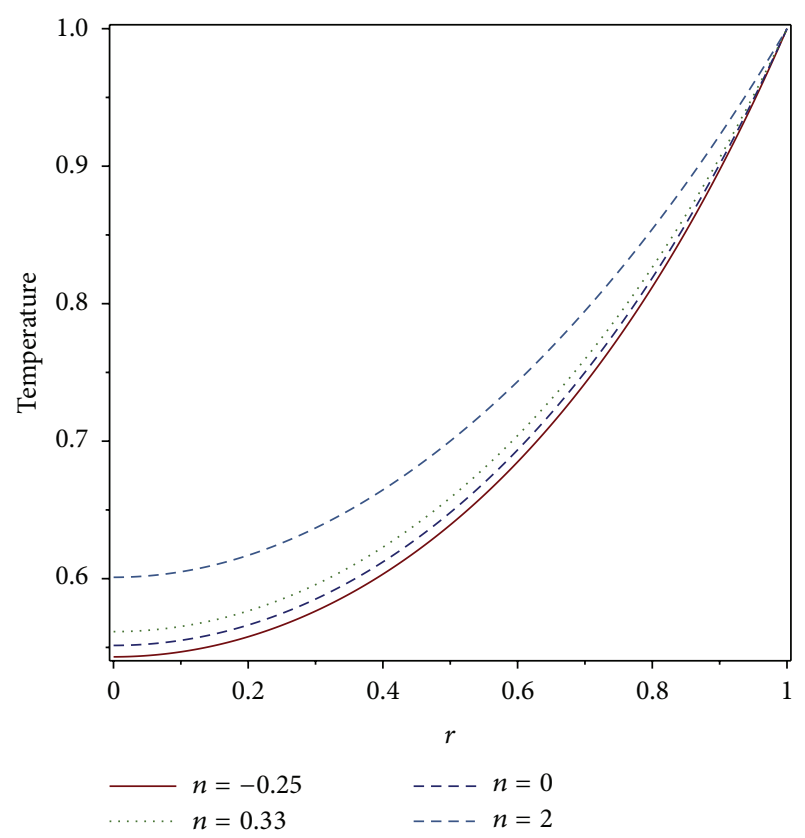

FIGURE 6: Temperature profile given solutions (17) for varying values of $n$. Here $M=2$ is fixed.

Case 4 ( $\alpha=$ arbitrary, $n<-1$ and $n>-1)$. Given an arbitrary $\alpha$, we obtain the general solutions

$$
\begin{gathered}
\theta=\left\{r ^ { 1 / 2 - \alpha / 2 } \left[c_{1} J_{\alpha / 2-1 / 2}(M \sqrt{n+1} r)\right.\right. \\
\left.\left.+c_{2} Y_{\alpha / 2-1 / 2}(M \sqrt{n+1} r)\right]\right\}^{1 /(n+1)}, \\
n<-1, \\
\theta=\left\{r ^ { 1 / 2 - \alpha / 2 } \left[c_{1} J_{\alpha / 2-1 / 2}(i M \sqrt{n+1} r)\right.\right. \\
\left.\left.+c_{2} Y_{\alpha / 2-1 / 2}(i M \sqrt{n+1} r)\right]\right\}^{1 /(n+1)}, \\
n>-1 .
\end{gathered}
$$

Note that one may obtain exact solutions which satisfy the boundary conditions only when $\alpha=1$ but this will coincide with solution (16). One may also construct exact solution when $m \neq n=-1$. In this case the solution satisfying the boundary condition is given by

$$
\theta=(m+1)\left[\frac{M^{2} r^{2}}{2(\alpha+1)}+\frac{1}{m+1}-\frac{M^{2}}{2(\alpha+1)}\right]
$$

The solution (20) is depicted in Figure 7.

\section{Symmetry Reductions}

In this section we consider the case $m \neq n$, with $\alpha=2$ and $\alpha$ being arbitrary. In this case (4) is not linearizable and as such we employ the Lie point symmetry analysis.

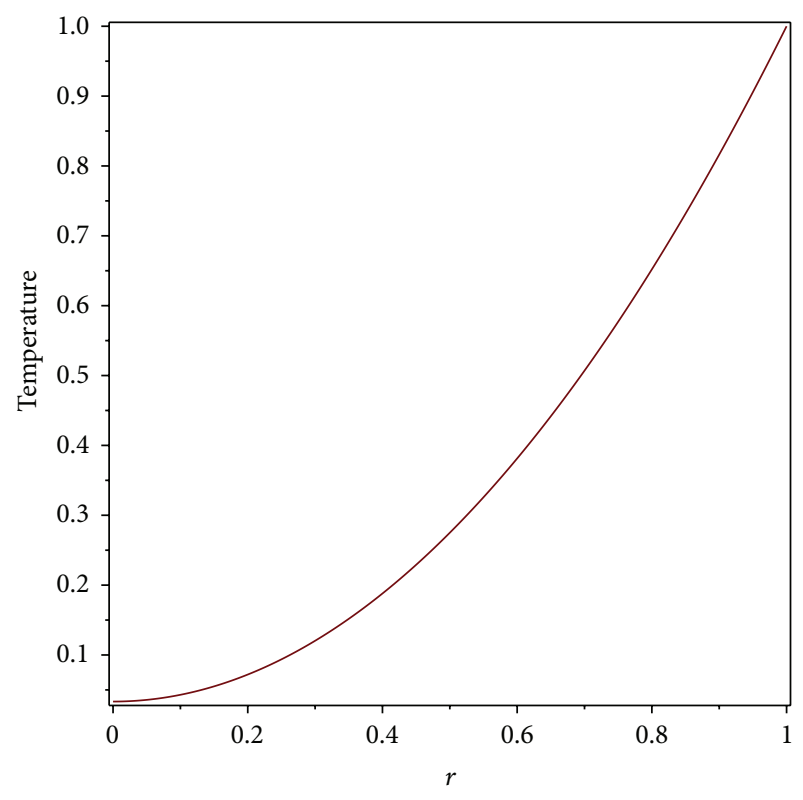

FIGURE 7: Temperature profile given solutions (20) for varying values of $n$. Here $M=2$ is fixed.

5.1. Case $\forall \alpha \neq 0$. In this case (4) admits one-dimensional Lie algebra spanned by the base vector

$$
X=\frac{m}{2(m+1)}\left(-2 \theta \frac{\partial}{\partial \theta}+(n-m) r \frac{\partial}{\partial r}\right) .
$$

Note that an obvious extra symmetry when $\alpha=0$ is a translation in $r$. We employ method of differential invariants to reduce the order of (4) by one. The first prolongation of the generator $X$ is given by

$$
\begin{aligned}
X^{[1]}= & \frac{m}{2(m+1)}\left(-2 \theta \frac{\partial}{\partial \theta}+(n-m) r \frac{\partial}{\partial r}\right) \\
& -\left[\frac{2 m+m(n-m)}{2(m+1)}\right] \theta^{\prime} \frac{\partial}{\partial \theta^{\prime}},
\end{aligned}
$$

and the corresponding characteristic equation is given by

$$
\frac{d r}{m(n-m) r}=-\frac{d \theta}{2 m \theta}=-\frac{d \theta^{\prime}}{[2 m+m(n-m)] \theta^{\prime}} .
$$

Solving the above characteristics gives the invariants

$$
I_{1}=\theta r^{2 /(n-m)}, \quad I_{2}=\theta^{\prime} r^{(2+n-m) /(n-m)} .
$$

Now we let $I_{1}=t, I_{2}=u$ and write $u=u(t)$. From the definition of $t$ and by using chain rule

$$
D_{r}=D_{r}(t) D_{t}
$$

we obtain

$$
\frac{2+n-m}{n-m} \theta^{\prime}+r \theta^{\prime \prime}=\left[\frac{2 \theta}{(n-m) r}+\theta^{\prime}\right] u^{\prime} .
$$


Substituting into (8) we have

$$
u^{\prime}\left(\frac{2 t^{2}}{n-m}+t u\right)+\alpha t u+m u^{2}-M^{2} t^{n-m+2}=0 .
$$

We notice that the above equation may not be solved exactly.

5.1.1. Subcase $\alpha=(3 m+n+4) /(m+n+2)$. This choice of $\alpha$ is not physical since there is no relationship between the geometry of the fin and the exponents of the thermal conductivity and heat transfer coefficient. However, this case is mathematically interesting since (4) admits two Lie point symmetries which implies [18] that the ODE in question is integrable or reducible to the one with cubic degree in first derivative. The admitted Lie algebra is spanned by the base vectors

$$
\begin{gathered}
X_{1}=\frac{1}{m-n}\left(2 \theta \frac{\partial}{\partial \theta}+(m-n) r \frac{\partial}{\partial r}\right), \\
X_{2}=\exp \left(\frac{n+1}{m+n+2}\right) \\
\times r^{2}\left[2(m+n+2) \theta \frac{\partial}{\partial \theta}+(n+2)(2 m+n+2) r \frac{\partial}{\partial r}\right] .
\end{gathered}
$$

We omit further analysis since the initial assumption is not physically realistic.

5.1.2. Subcase $\alpha=2, n=-1$. In this subcase (4) is integrable and admits an eight-dimensional Lie algebra spanned by the base vectors

$$
\begin{aligned}
& X_{1}=-\frac{r^{2} \theta^{-m}}{3}\left(M^{2} r \frac{\partial}{\partial \theta}+3 \theta^{m} \frac{\partial}{\partial r}\right), \\
& X_{2}=-\frac{2 r \theta^{-m}}{3}\left(M^{2} r \frac{\partial}{\partial \theta}+3 \theta^{m} \frac{\partial}{\partial r}\right), \\
& X_{3}=\frac{m}{2(m+1)} \\
& \times\left\{\left[(m+1) M^{2} r^{2}-2 \theta^{m+1}\right] \frac{\partial}{\partial \theta}+2(m+1) r \theta^{m} \frac{\partial}{\partial r}\right\}, \\
& X_{4}=\frac{r^{2} \theta^{-m}}{18(m+1)}\left\{\left(m M^{4} r+M^{2} r^{3}-6 M^{2} r \theta^{m+1}\right) \frac{\partial}{\partial \theta}\right. \\
& \left.+\left[3 M^{2}(m+1) \theta^{m}-18 \theta^{2 m+1}\right] \frac{\partial}{\partial r}\right\}, \\
& X_{5}=\frac{\theta^{-m}}{12(m+1)^{2}} \\
& \times\left\{\left[12 \theta^{2(m+1)}-8(m+1) M^{2} r^{2} \theta^{m+1}\right.\right. \\
& \left.+M^{4}(m+1)^{2} r^{4}\right] \frac{\partial}{\partial \theta} \\
& +\left[2(m+1)^{2} M^{2} r^{3} \theta^{m}-12(m+1) r \theta^{2 m+1}\right] \\
& \left.\times \frac{\partial}{\partial r}\right\} \text {, }
\end{aligned}
$$

$$
\begin{aligned}
& X_{6}=\frac{m\left[(m+1) M^{2} r^{2}-6 \theta^{m+1}\right]}{6(m+1) \theta^{m}} \frac{\partial}{\partial \theta}, \\
& X_{7}=-\frac{\theta^{-m}}{r} \frac{\partial}{\partial \theta}, \quad X_{8}=-\theta^{-m} \frac{\partial}{\partial \theta} .
\end{aligned}
$$

Equation (4) is equivalent to the simple motion equation $y^{\prime \prime}=0$ [18]. We adopt method of canonical coordinate to demonstrate this claim. We introduce the method of finding the solutions using $X_{7}$ and $X_{8}$ from the above dimensional Lie algebra vectors. The two symmetries lead to the canonical variables

$$
t=r, \quad u=-\frac{r \theta^{1+m}}{1+m},
$$

and the corresponding canonical forms of $X_{7}$ and $X_{8}$ are

$$
X_{1}=\partial_{u}, \quad X_{2}=t \partial_{u} .
$$

Writing $u=u(t)$ transforms (4) into

$$
u^{\prime \prime}(t)+t M^{2}=0 .
$$

Integrating the latter equation and writing it into its original variables we obtain

$$
\theta(r)=\left\{\left[\frac{1}{6} M^{2} r^{2}-\frac{c_{1}}{r}-c_{2}\right](1+m)\right\}^{1 /(1+m)} .
$$

Imposing the boundary conditions, we obtain

$$
\theta=r^{2 /(m+1)}, \quad \forall m<1 .
$$

5.1.3. Subcase $\alpha=2, m=n$. In this subcase the admitted eight-dimensional Lie symmetry algebra includes

$$
\begin{aligned}
& X_{1}=\theta \frac{\partial}{\partial \theta}, \\
& X_{2}=\frac{\partial}{\partial r}-\frac{\theta}{(1+n) r} \frac{\partial}{\partial \theta}, \\
& X_{3}=\frac{e^{-\sqrt{M^{2}(1+n)} r} \theta^{-n}}{r} \frac{\partial}{\partial \theta}, \\
& X_{4}=\frac{e^{\sqrt{M^{2}(1+n)} r} \theta^{-n}}{\sqrt{M^{2}(1+n)} r} \frac{\partial}{\partial \theta}, \\
& X_{5}=(1+n) M^{2} \frac{\partial}{\partial r}+\left(M^{3} \sqrt{1+n}-\frac{M^{2}}{r}\right) \theta \frac{\partial}{\partial \theta}, \\
& X_{6}=-(1+n) M^{2} \frac{\partial}{\partial r}+\left(M^{3} \sqrt{1+n}+\frac{M^{2}}{r}\right) \theta \frac{\partial}{\partial \theta}, \\
& X_{7}=(1+n) \frac{\partial}{\partial r}-\left(1+\sqrt{M^{2}(1+n) r}\right) \theta \frac{\partial}{\partial \theta}, \\
& X_{8}=(1+n) \frac{\partial}{\partial r}-\left(1-\sqrt{M^{2}(1+n) r}\right) \theta \frac{\partial}{\partial \theta} .
\end{aligned}
$$


Since (4) admits eight symmetries it is linearizable (see also [18]). Using the symmetry generators $X_{1}$ and $X_{2}$, we obtain the canonical variables

$$
t=\ln \left(\theta r^{1 /(1+n)}\right), \quad u=r .
$$

The corresponding generators in canonical variables are

$$
X_{1}=\partial_{t}, \quad X_{2} \partial_{u} .
$$

Writing $u=u(t)$ transforms (4) into

$$
u^{\prime \prime}=(n+1) u^{\prime}-M^{2} u^{\prime 3} .
$$

\section{Fin Efficiency and Heat Flux}

The fin efficiency is defined as the ratio of the actual heat transfer from the fin surface to the surrounding fluid while the whole fin is kept at the same temperature. On the other hand, heat flux is the total amount of heat flowing per unit area per unit time. The fin efficiency and the heat flux in dimensionless variables are given by

$$
\begin{aligned}
& \eta=\int_{0}^{1} \theta^{n+1} d r, \\
& q=\frac{1}{B i} \frac{k(\theta)}{h(\theta)} \frac{d \theta}{d r},
\end{aligned}
$$

respectively. Here the dimensionless parameter $B i=h_{b} L / k_{a}$ is the Biot number.

Given solution (17), we obtain

$$
\begin{aligned}
\eta= & (\ln (M \sqrt{n+1})-\ln (-M \sqrt{n+1}) \\
& +E i(1, M \sqrt{n+1})-E i(1,-M \sqrt{n+1})) \\
\times & (2 \sinh (M \sqrt{n+1}))^{-1},
\end{aligned}
$$

where $\operatorname{Ei}(a, z)$ is the exponential integral [19]. Fin efficiency (41) is depicted in Figure 8.

And the heat flux becomes

$$
q=\frac{1}{B i} \frac{r M \sqrt{n+1} \cosh (M \sqrt{n+1} r)-\sinh (M \sqrt{n+1} r)}{(n+1) r \sinh (M \sqrt{n+1} r)}
$$

Heat flux (42) is depicted in Figures 9 and 10.

\section{Some Discussions and Concluding Remarks}

In Figure 4, we observe that heat is transferred much slower in spherical fins than in radial and rectangular fins. This is also confirmed by the values in Table 1 . Now we focus only on spherical fins and observe in Figure 5 that temperature decreases with increase in the values of $M$. Recall that the thermogeometric fin parameter is directly proportional to the aspect ratio of the fin. Thus longer fins ( $M$ larger) release heat much more efficiently that shorter ones. In Figure 6,

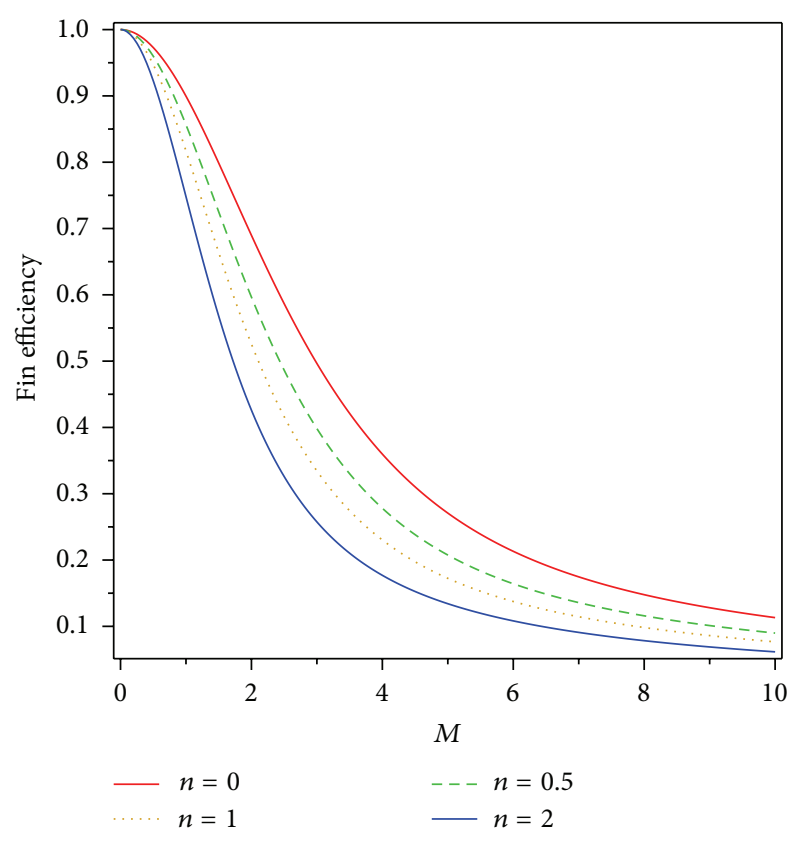

FIGURE 8: Fin efficiency for varying values of $n$.

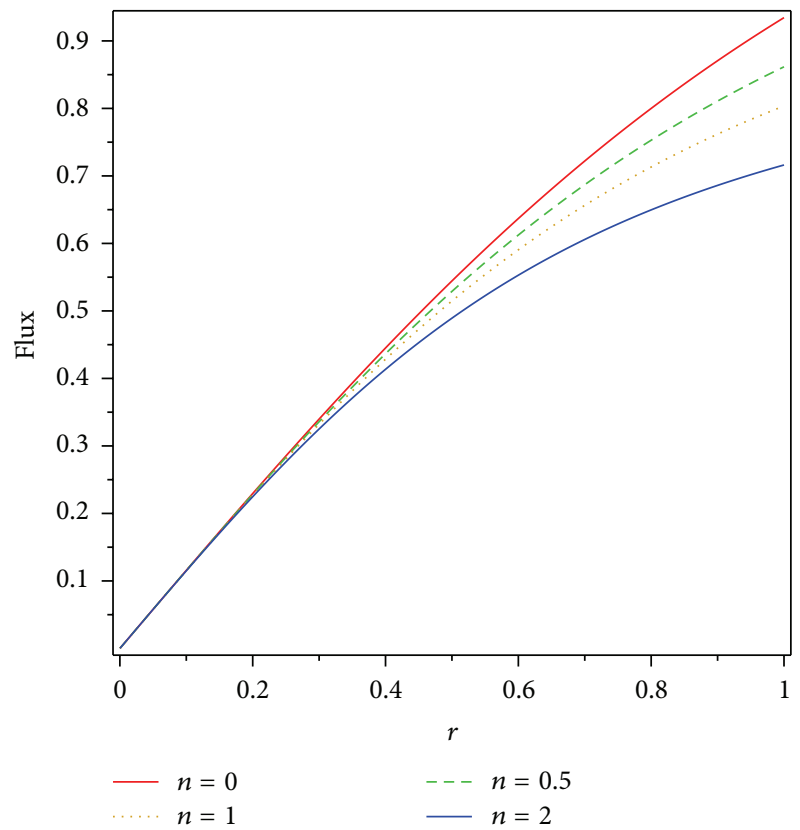

FIGURE 9: Heat flux for varying values of $n$.

temperature increases with an increase in the values of $n$. Finally Figure 7 depicts the heat transfer where $m \neq n=1$. Figure 8 is a plot of the fin efficiency with varying values of $n$. Figures 9 and 10 depict the heat flux. In this paper we focused on the comparison of temperature distribution (or heat transfer) in fins of different geometries. One may observe from Table 1 that at any given point $r_{0}$ on the radius $r$ the temperature values are much higher in a spherical fin than radial and rectangular geometries. It turns out that the spherical fin is not as effective in transferring heat as the radial or rectangular fins. 


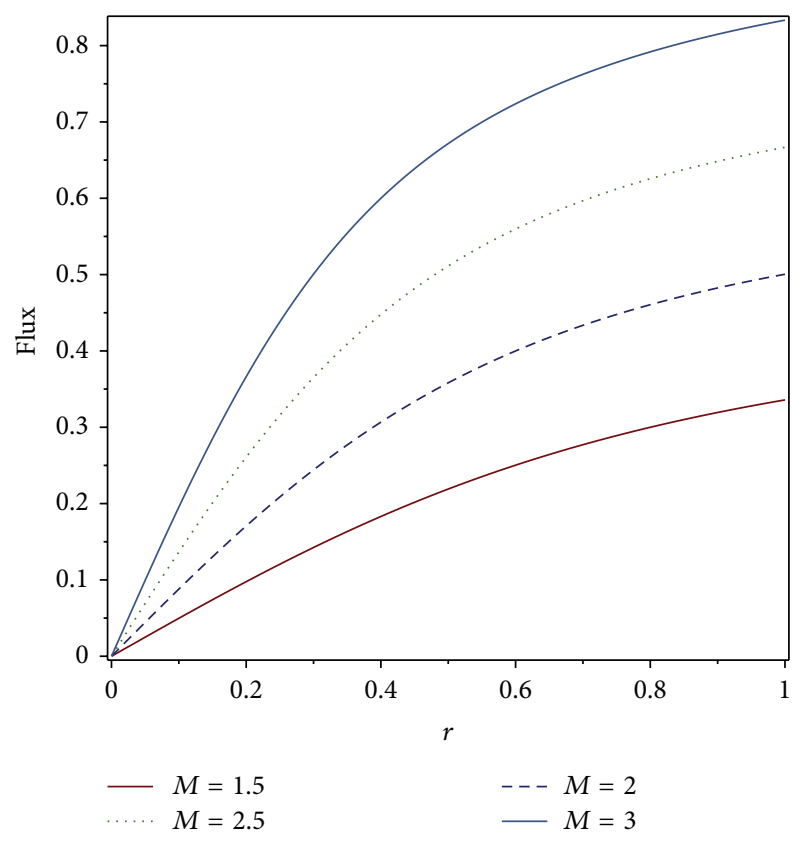

FIGURE 10: Heat flux for varying values of $M$.

TABle 1: Comparison of the temperature values in fins with rectangular, radial, and spherical profiles. Here $M=4$ and $n=1$.

\begin{tabular}{lccc}
\hline$r$ & $\begin{array}{c}\alpha=0 \\
\text { (rectangular) }\end{array}$ & $\begin{array}{c}\alpha=1 \\
\text { (radial) }\end{array}$ & $\begin{array}{c}\alpha=2 \\
\text { (spherical) }\end{array}$ \\
\hline 0 & 0.125136 & 0.185069 & 0.238556 \\
0.1 & 0.13479 & 0.192403 & 0.244902 \\
0.2 & 0.160849 & 0.213754 & 0.363795 \\
0.3 & 0.199607 & 0.248095 & 0.295078 \\
0.4 & 0.25049 & 0.295331 & 0.33908 \\
0.5 & 0.315255 & 0.356686 & 0.396937 \\
0.6 & 0.397056 & 0.434579 & 0.470685 \\
0.7 & 0.500174 & 0.532528 & 0.563297 \\
0.8 & 0.630101 & 0.655185 & 0.678749 \\
0.9 & 0.793788 & 0.808503 & 0.822159 \\
1 & 1 & 1 & 1 \\
\hline
\end{tabular}

\section{Conflict of Interests}

The authors declare that there is no conflict of interests regarding the publication of this paper.

\section{Acknowledgment}

R. J. Moitsheki is grateful to the National Research Foundation of South Africa for the generous financial support.

\section{References}

[1] M. Pakdemirli and A. Z. Sahin, "Group classification of fin equation with variable thermal properties," International Journal of Engineering Science, vol. 42, no. 17-18, pp. 1875-1889, 2004.
[2] A. H. Bokhari, A. H. Kara, and F. D. Zaman, "A note on a symmetry analysis and exact solutions of a nonlinear fin equation," Applied Mathematics Letters, vol. 19, no. 12, pp. 13561360, 2006.

[3] M. Pakdemirli and A. Z. Sahin, "Similarity analysis of a nonlinear fin equation," Applied Mathematics Letters, vol. 19, no. 4, pp. 378-384, 2006.

[4] O. O. Vaneeva, A. G. Johnpillai, R. O. Popovych, and C. Sophocleous, "Group analysis of nonlinear fin equations," Applied Mathematics Letters, vol. 21, no. 3, pp. 248-253, 2008.

[5] R. O. Popovych, C. Sophocleous, and O. O. Vaneeva, "Exact solutions of a remarkable fin equation," Applied Mathematics Letters, vol. 21, no. 3, pp. 209-214, 2008.

[6] F. Khani, M. A. Raji, and H. Hamedi-Nejad, "Analytical solutions and efficiency of the nonlinear fin problem with temperature-dependent thermal conductivity and heat transfer coefficient," Communications in Nonlinear Science and Numerical Simulation, vol. 14, no. 8, pp. 3327-3338, 2009.

[7] R. J. Moitsheki, T. Hayat, and M. Y. Malik, "Some exact solutions of the fin problem with a power law temperature-dependent thermal conductivity," Nonlinear Analysis, Real World Applications, vol. 11, no. 5, pp. 3287-3294, 2010.

[8] R. J. Moitsheki, "Steady heat transfer through a radial fin with rectangular and hyperbolic profiles," Nonlinear Analysis: Real World Applications, vol. 12, no. 2, pp. 867-874, 2011.

[9] R. J. Moitsheki, "Steady one-dimensional heat flow in a longitudinal triangular and parabolic fin," Communications in Nonlinear Science and Numerical Simulation, vol. 16, no. 10, pp. 3971-3980, 2011.

[10] R. J. Moitsheki and M. D. Mhlongo, "Classical lie point symmetry analysis of a steady nonlinear one-dimensional fin problem," Journal of Applied Mathematics, vol. 2012, Article ID 671548, 13 pages, 2012.

[11] P. L. Ndlovu and R. J. Moitsheki, "Analytical solutions for steady heat transfer in longitudinal fins with temperature dependent properties," Mathematical Problems in Engineering, vol. 2013, Article ID 273052, 14 pages, 2013.

[12] A. Moradi, "Analytical solutions for fin with temperature dependant heat transfer coefficient," International Journal of Engineering \& Applied Sciences, vol. 3, no. 2, pp. 1-12, 2011.

[13] G. W. Bluman and S. Kumei, Symmetries and Differential Equations, vol. 81 of Applied Mathematical Sciences, Springer, New York, NY, USA, 1989.

[14] G. W. Bluman and S. Anco, Symmetry and Integration Methods for Differential Equations, vol. 154 of Applied Mathematical Sciences, Springer, New York, NY, USA, 2002.

[15] G. W. Bluman, A. Cheviakov, and S. Anco, Applications of Symmetry Methods to Partial Differential Equations, vol. 168 of Applied Mathematical Sciences, Springer, New York, NY, USA, 2010.

[16] P. J. Olver, Applications of Lie Groups to Differential Equations, vol. 107 of Graduate Texts in Mathematics, Springer, New York, NY, USA, 1986.

[17] N. H. Ibragimov, Elementary Lie Group Analysis and Ordinary Differential Equations, vol. 4 of Mathematical Methods in Practice, John Wiley \& Sons, Chichester, UK, 1999.

[18] F. M. Mahomed, "Symmetry group classification of ordinary differential equations: survey of some results," Mathematical Methods in the Applied Sciences, vol. 30, no. 16, pp. 1995-2012, 2007.

[19] M. Abramowitz and I. A. Stegun, Handbook of Mathematicsl Functions, Dover, New York, NY, USA, 1972. 


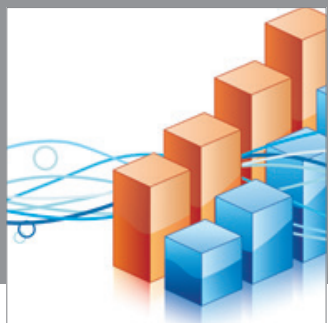

Advances in

Operations Research

mansans

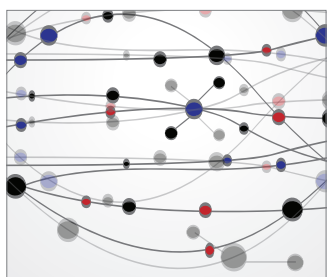

The Scientific World Journal
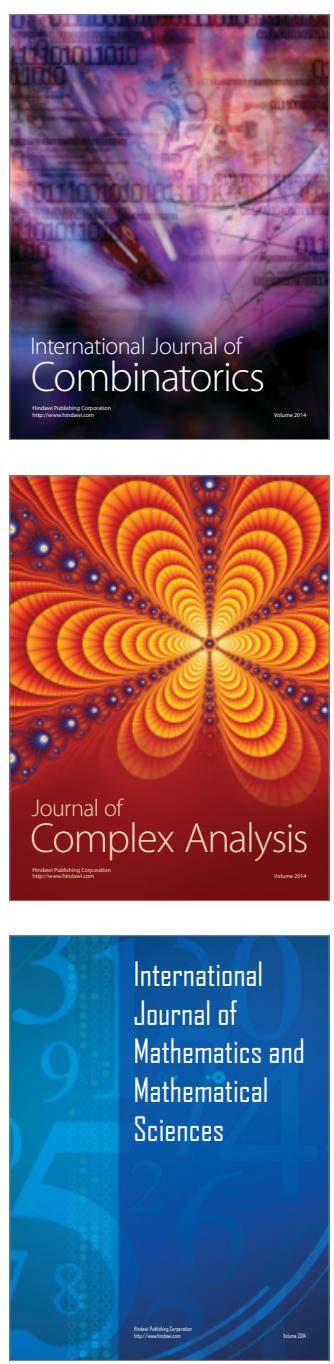
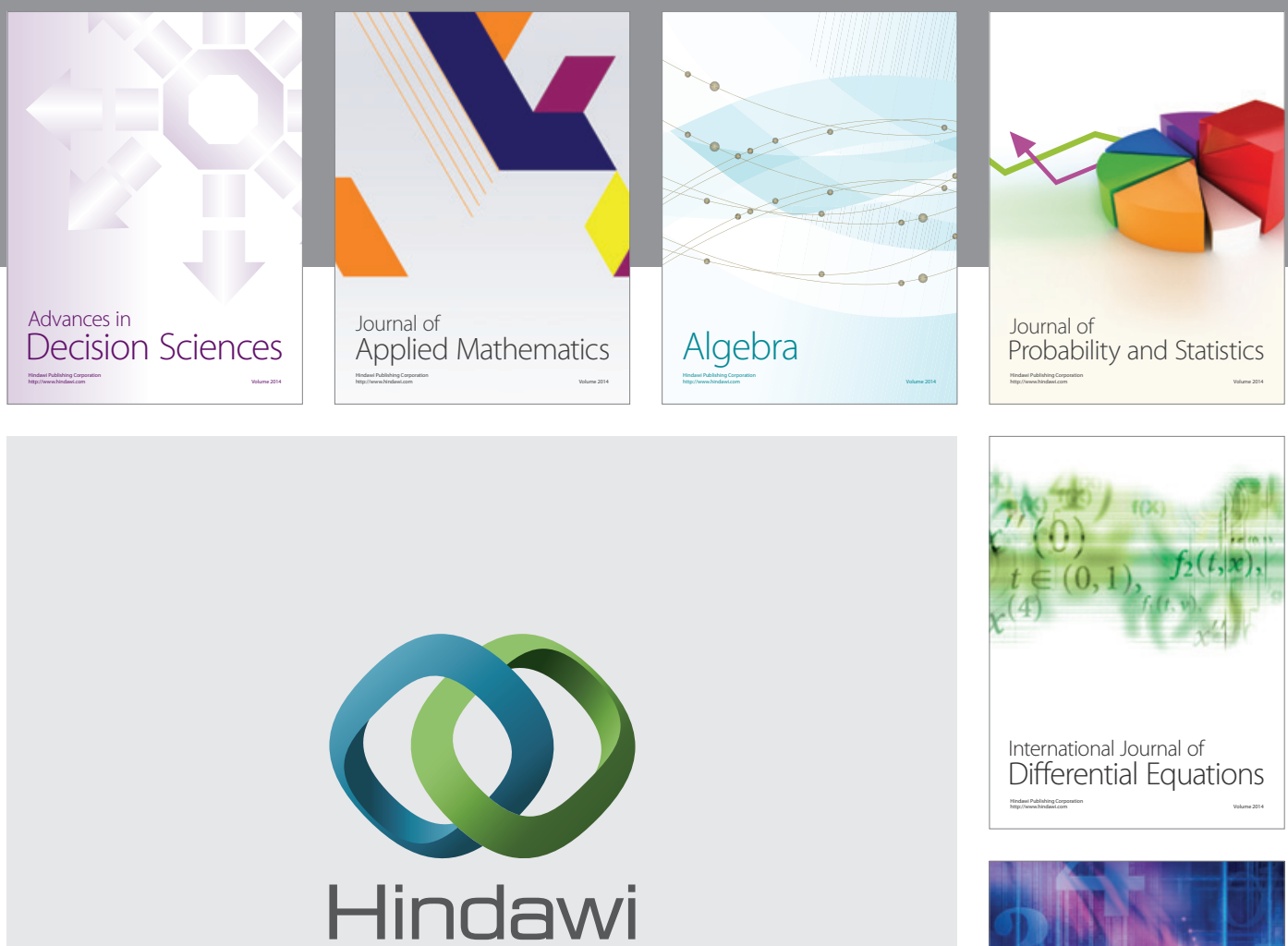

Submit your manuscripts at http://www.hindawi.com
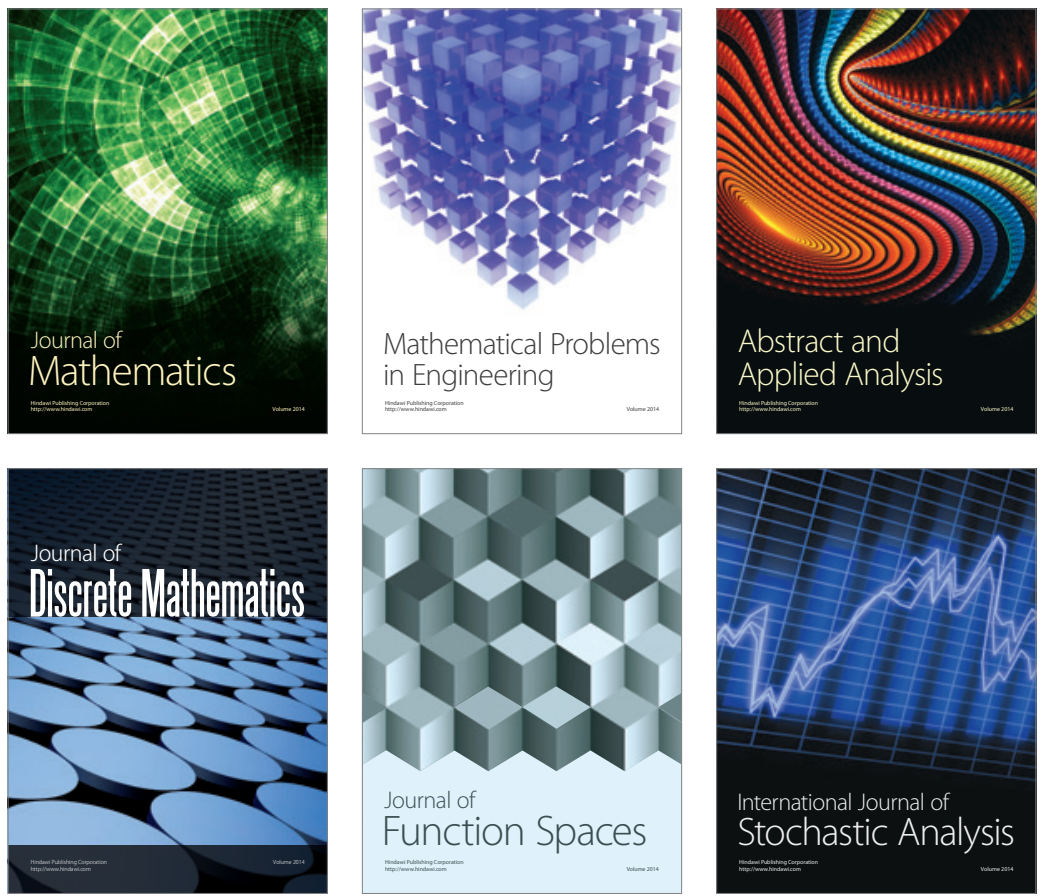

Journal of

Function Spaces

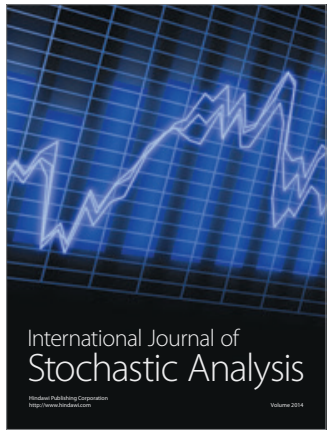

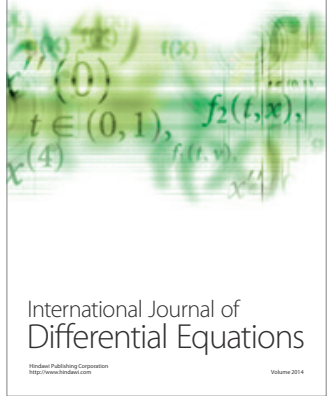
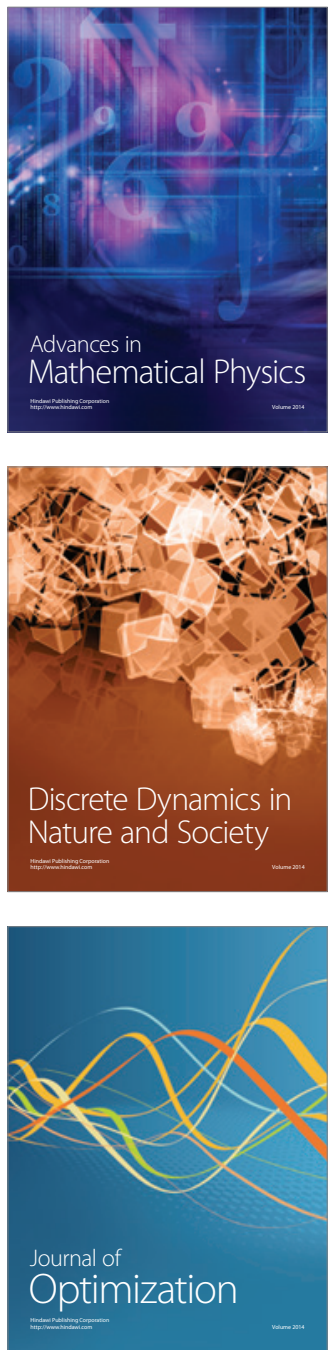